Pacific Journal of Mathematics

COMPLETE REDUCIBILITY OF ADMISSIBLE 


\title{
COMPLETE REDUCIBILITY OF ADMISSIBLE REPRESENTATIONS OVER FUNCTION FIELDS
}

\author{
STEPhen J. HARIS
}

In his investigations of the "natural domain of validity" for all Siegel Formulae over number fields, Igusa was lead to a certain class of representations, which however, make sense over any field, not just number fields. Calling these representations absolutely admissible, Igusa analyzed their arithmetic nature in "Geometry of absolutely admissible representations" [4], to find the ring of invariants, the stabilizers of various points etc. The objective of [2] and of this paper is to show that for function fields, the absolutely admissible representations arise from the same arithmetic questions concerning the Siegel Formula, as was the case for number fields. In [2] we obtained a list of the composition factors of the representations that arise in this manner. In the present paper we show that these representations are in fact completely reducible, whence for the characteristic of the function field sufficiently large (a bound given explicitly for each group) the arithmetic of invariants discussed in [4] hold for function fields, exactly as for number fields.

The method of proof is cohomological, using the structure theory of semi-simple groups to find a sufficient condition, which will guarantee that the extensions split. A case by case examination shows that this condition is satisfied in every case save for $S L_{2}$ and $E_{6}$, where further arguments are needed.

The author wishes to thank John Sullivan for helpful conversations concerning the cohomology.

1. A sufficient condition for extensions to split. Let $G$ be a linear algebraic group defined over a field $k$ and fix a universal domain $\Omega \supset k$. Let $V, W$ be two rational $G$-modules that are finite dimensional vector spaces over $\Omega$ and set $W^{*}=\operatorname{Hom}_{\Omega}(W, \Omega)$. By the theory of derived functors we can identify $\operatorname{Ext}_{G}(V, W)$ with $H\left(G, V \otimes_{\Omega} W^{*}\right)$, since the category of $G$-modules has enough injectives [3]. In particular $\operatorname{Ext}_{G}^{1}(V, W) \cong H^{1}\left(G, V \otimes_{\Omega} W^{*}\right)$, whence the extension of $W$ by $V$ splits if $H^{1}\left(G, V \otimes_{\Omega} W^{*}\right)=0$.

Proposition 1. Let $G$ be a semi-simple algebraic group, $V$ a rational G-module, which is a finite dimensional vector space over Q. Fix a maximal torus $T$ of $G$ and a Borel subgroup $B \supset T$, 
which will serve to order the roots of $G$. If no weight of $G$ in $V$ is of the form $\pm a \alpha$, where $a \geqq 1$, integer, $\alpha$ a simple root of $G$, then $H^{1}(G, V)=0$.

Proof. Let $P_{\alpha}$ be the one parameter subgroup associated with the root $\alpha$, i.e., the image of the morphism $x_{\alpha}: G_{\alpha} \rightarrow G$, where $t x_{\alpha}(z) t^{-1}=x_{\alpha}\left(t^{\alpha} z\right)$. Here $t \in T, \alpha$ is a root of $G$ (with respect to $T$ ), $z \in \Omega$ and $t^{\alpha}=\alpha(t)$. Then $G$ is generated by $T, P_{\alpha}, P_{-\alpha}$ for $\alpha \in \Delta$, the simple roots for the ordering determined by $B$. Let $f: G \rightarrow V$ be a 1-cocycle. Since the cohomology groups of tori are trivial, we can modify $f$ by a suitable coboundary to have $f(t)=0$, for all $t \in T$. Now $V=\sum_{s} V_{s}$, where $V_{s}=\left\{v \in V \mid t . v=t^{s} v\right.$, all $\left.t \in T\right\}$. So, writing $f\left(x_{\alpha}(z)\right)=\sum_{s} l_{s}(z)$, where $z \rightarrow l_{s}(z)$ is a regular function $G_{a} \rightarrow V_{s}$, since it is given by a polynomial function, we have $f\left(x_{\alpha}(z)\right)=\sum_{i \geq 0, s} z^{i} l_{s, i}$ with $l_{s, i} \in V_{s}$. But $f(i d)=0$, so in fact $f\left(x_{\alpha}(z)\right)=\sum_{i \geqq 1, s} z^{i} l_{s, i}$.

Now, using the cocycle relation $f\left(g g^{\prime}\right)=f(g)+g \cdot f\left(g^{\prime}\right)$ and the conjugation relation $t x_{\alpha}(z) t^{-1}=x_{\alpha}\left(t^{\alpha} z\right)$ we see upon comparing the coefficients of $z^{i}$ that if $l_{s, i} \neq 0$, then $t^{\alpha i}=t^{s}$, so if the weights of $G$ in $V$ are not of the form $\pm i \alpha, i \geqq 1 \alpha \in \Delta$ we must have $f=0$, whence $H^{1}(G, V)=0$.

Corollary. If $V, W$ are $G$-modules, then the extension of $W$ by $V$ splits if the weights of $V \otimes W^{*}$ are not of the form $\pm i \alpha$ for some $i \geqq 1, \alpha \in \Delta$.

Further, every $G$-module all of whose composition factors have the same highest weight $\lambda$, is completely reducible, for $G$ semi-simple. This is a consequence of the fact that for an irreducible representation there is a unique Borel stable line, the multiplicity of $\lambda$ is one and every other weight is of the form $s=\lambda-\sum_{\alpha \in \Delta} m_{\alpha}(s) \alpha$, with $m_{\alpha}(s) \geqq 0$ an integer [1]. We shall give a proof of this known result because of its importance in our work and for lack of a convenient reference.

LemMa (Humphreys). G a semi-simple group, $V$ a rational $G$ module all of whose composition factors have the same highest weight. Then $V$ is completely reducible.

Proof. For $y \in V_{s}, x_{\alpha}(z) \cdot y=y+$ vectors of weights $s+i \alpha$ $(i>0)$. This can be seen exactly as in Proposition 1; hence if $\lambda$ is the highest weight that occurs in $V$, every $v \in V_{\lambda}$ gives rise to a Borel stable line, since vectors of weight $\lambda+i \alpha(i>0, \alpha>0)$ cannot occur. Also $\operatorname{span}(G . v)=\operatorname{span}\left(U^{-} . v\right)$ for such $v \in V_{\lambda}$, so this has a 
unique line of weight $\lambda$, whence $\operatorname{span}(G . v)$ is an irreducible $G$ module, since any $G$-submodule must have a Borel stable line, while the only highest weights in $V$ are $\lambda$, thus such a line must contain $v$, by the uniqueness just remarked. Let $W$ be the $G$-submodule of $V$ generated by the vectors of weight $\lambda$. Then $W=\left\langle G \cdot v_{1}\right\rangle+\cdots+$ $\left\langle G . v_{r}\right\rangle$ is completely reducible. If $W \neq V$, then $V \cong W+V / W$ as $T$-module, the highest weight that can occur in $V / W$ is less than $\lambda$, contrary to $V$ having only $\lambda$ as highest weight of the composition factors.

The irreducible representations of $G$ are characterized by their highest weight and for a given $\lambda$, the irreducible $G$-module (in characteristic $P$ ) having highest weight $\lambda$ is a quotient of the corresponding module (in characteristic 0 ). Thus, to obtain complete reducibility, it suffices to show that for the modules in characteristic 0 the weights are not of the form $\pm a \alpha$. By the above lemma, we need only consider the composition factors corresponding to different weights.

2. Complete reducibility of admissible representations. From [2] and the previous remarks, we need to investigate the following representations.

Type $A_{n} . \rho_{1}+\rho_{n}, \rho_{1}+\rho_{2}, \rho_{1}+\rho_{n-1}, \rho_{2}+\rho_{n}, \rho_{n-1}+\rho_{n}, \rho_{P} a_{A_{1}}+$ ${ }_{P} b_{\Lambda_{n}}$. By the outer automorphism of the Dynkin diagram we have $\check{\rho}_{i}=\rho_{n-i+1}$ where $\check{\rho}$ denotes the contragredient representation.

Case $\rho_{i}+\rho_{n}$. $\rho_{1} \otimes \check{\rho}_{n}=\rho_{1} \otimes \rho_{1}$. The simple roots are $\lambda_{1}-\lambda_{2}$, $\lambda_{2}-\lambda_{3}, \cdots, \lambda_{n}-\lambda_{n+1}$, while the weights of $\lambda_{1}$ are $\lambda_{1}, \lambda_{2}, \cdots, \lambda_{n}$, $\lambda_{n+1}=\left(\lambda_{1}+\cdots+\lambda_{n}\right)$, whence the weights $\rho_{1} \otimes \rho_{1}$, being $\lambda_{i}+\lambda_{j}$ are never of the form $\pm a\left(\lambda_{i}-\lambda_{i+1}\right)$ if $n \geqq 2$.

For $n=1, \alpha=2 \lambda_{1}$ and the weights of $\rho_{1} \otimes \rho_{1}$ are $\pm 2 \lambda_{1}, 0$, so a further argument is needed. But for $f: S L_{2} \rightarrow V \otimes V$ a 1-cocycle, modified so that $\left.f\right|_{T}=0$, we have $t . f\left(x_{\alpha}(z)\right)=f\left(x_{\alpha}\left(t^{\alpha} z\right)\right)$, so if $V=$ $\left\langle v_{1}, v_{2}\right\rangle, f\left(x_{\alpha}(z)\right)=l_{1}(z) v_{1} \otimes v_{1}+l_{2}(z) v_{1} \otimes v_{2}+l_{3}(z) v_{2} \otimes v_{1}+l_{4}(z) v_{2} \otimes v_{2}$. Then the above relation implies that $f\left(x_{\alpha}(z)\right)=a z\left(v_{1} \otimes v_{1}\right), f\left(x_{-a}(z)\right)=$ $b z\left(v_{2} \otimes v_{2}\right)$ for constants $a, b$.

The cocycle relation applied to the element $x_{\alpha}(z) x_{-\alpha}\left(z^{\prime}\right)$ when $z z^{\prime}+1 \neq 0$, now implies that $a=b=0$, whence $H^{1}\left(S L_{n}, \rho_{1} \otimes \rho_{1}\right)=0$ for all $n$.

Case $\rho_{1}+\rho_{2}$. The analysis is similar to the above, since the weights of $\rho_{2}$ are known to be $\lambda_{i}+\lambda_{j}(i \neq j), \check{\rho}_{2}=\rho_{n-1}$. 
Case $\rho_{p^{a} A_{1}+p^{b} A_{n}} \cdot \rho_{p^{a} \Lambda_{1}} \otimes \check{\rho}_{p^{b} \Lambda_{n}}$ has weights $p^{a+b}$ (weights of $\rho_{1} \otimes \rho_{1}$ ), whence for $n \neq 1$, we have complete reducibility. But by [2], $n=1$ cannot occur, hence this case by case examination shows that every one of the representations known to be admissible for $S L_{n}$ is completely reducible. As we know this listing is exhaustive for $p>$ $3 / 2 n(n+1)$.

Type $B_{n}$. For $G=\operatorname{Spin}_{2 n+1}$, we must show $\rho_{1}+\rho_{n}$ is completely reducible, for $n=2,3,4$. The point now is to obtain the weights of $\rho_{n}$, the spin representation. By Chevalley's theorem [6, p. 14], if $W$ is the Weyl group acting on $E$, with $F$ a subset of $E$, then $W_{F}$, the subgroup of $W$ which keeps $F$ pointwise fixed is generated by the reflections $W_{\alpha_{i}}$ which keeps $F$ pointwise fixed.

Lemma. The weights of $\rho_{n}$ are $\left\{1 / 2\left( \pm \lambda_{1} \pm \lambda_{2} \pm \cdots \pm \lambda_{n}\right)\right\}$.

Proof. In characteristic zero, $\rho_{n}$ is a representation of degree $2^{n}$. For $\Lambda_{n}$ the highest weight of $\rho_{n}, W$ the Weyl group, $W . \Lambda_{n}$, the orbit of $A_{n}$ has [ $W: W_{A_{n}}$ ] elements. By Chevalley's theorem, we have $W_{A_{n}} \subset W\left(A_{n-1}\right)$, the Weyl group of type $A_{n-1}$ whence

$$
\left|W . \Lambda_{n}\right| \geqq\left|W: W\left(A_{n-1}\right)\right|=2^{n} n ! / n !=2^{n},
$$

so that $W$ acts transitively on the weights of $\rho_{n}$. In particular $\rho_{n}$ is of degree $2^{n}$ in characteristic $p$ with the weights $\left\{W \cdot \Lambda_{n}\right\}=$ $\left\{1 / 2\left( \pm \lambda_{1} \pm \cdots \pm \lambda_{n}\right)\right\}$. Note that $\rho_{n}$ is self contragredient.

For $B_{n}$, the simple roots are $\left\{\lambda_{1}-\lambda_{2}, \cdots, \lambda_{n-1}-\lambda_{n}, \lambda_{n}\right\}$ with the weights of $\rho_{1}$ being $\pm \lambda_{i}$. Thus $\pm \lambda_{i}+1 / 2\left(\lambda_{1} \pm \cdots \pm \lambda_{n}\right)$ is never of the form $\pm \alpha \alpha, \alpha \in \Delta$, whence $H^{1}\left(\operatorname{Spin}_{2 n+1}, \rho_{1} \otimes \rho_{n}\right)=0$. Therefore all the representations of a group of type $B_{n}$ listed in [2] are completely reducible.

Type $C_{n}$. Now $G=\mathrm{Sp}_{2 n}$ and we must show $\rho_{1}+\rho_{2}$ to be completely reducible where $\rho_{1}$ is the standard realization of $G$ on $M_{2 n, 1}$ and $\rho_{2}$ is the representation on $M_{2 n, 1} \wedge M_{2 n, 1}$. Thus the weights of $\rho_{1}$ are $\pm \lambda_{i}$, for $\lambda_{2}$ they are $\pm \lambda_{i}, \lambda_{j}, 0$. But the simple roots are $\left\{\lambda_{i}-\lambda_{i+1} 1 \leqq i<n, 2 \lambda_{n}\right\}$ so we. see that $\rho_{1}+\rho_{2}$ is completely reducible.

Type $D_{n}$. Now $G=\operatorname{Spin}_{2 n}$ and we must show that the following are completely reducible.

$n=4: \quad \rho_{1}+\rho_{3}+\rho_{4}, \rho_{1}+\rho_{3}, \rho_{1}+\rho_{4}, \rho_{3}+\rho_{4}$

$n=5: \quad \rho_{1}+\rho_{2}, \rho_{1}+\rho_{4}, \rho_{1}+\rho_{5}$

$n=6: \rho_{1}+\rho_{5}, \rho_{1}+\rho_{6}$ in general and if characteristic of $k=2$, for 
$n=5: \rho_{2 A_{1}}+\rho_{4}, \rho_{2 \Lambda_{1}}+\rho_{5}$. Exactly as was the case for type $B_{n}$, we see that the Weyl group operates transitively on the weights of $\rho_{n-1}, \rho_{n}$ (resp.) and as $W\left(D_{n}\right)=S_{n} \cdot(\boldsymbol{Z} / 2 \boldsymbol{Z})^{n-1}$ with $\lambda_{i} \rightarrow \pm \lambda_{\imath}$ with an even number of negative signs, so that the weights are, for $\rho_{n-1}$ : $\left\{1 / 2\left( \pm \lambda_{1} \pm \lambda_{2} \pm \cdots \pm \lambda_{n}\right)\right.$ even number of negative signs $\}$, for $\rho_{n}$ : $\left\{1 / 2\left( \pm \lambda_{1} \pm \lambda_{2} \pm \cdots \pm \lambda_{n}\right) \mid\right.$ odd number of negative signs $\}$.

Therefore $\rho_{1} \otimes \breve{\rho}_{n-1}, \rho_{1} \otimes \breve{\rho}_{n}$ do not have weights of the form $\pm \alpha \alpha, \quad \alpha \in\left\{\lambda_{i}-\lambda_{i+1} 1 \leqq i<n, \lambda_{n-1}+\lambda_{n}\right\}$, whence $\rho_{1}+\rho_{n-1}, \rho_{1}+\rho_{n}$ are completely reducible for all $n$. Also by the above, for $n=4$, $\rho_{3} \otimes \rho_{4}$ has weights $1 / 2\left( \pm \lambda_{1} \pm \lambda_{2} \pm \lambda_{3} \pm \lambda_{4}\right)+1 / 2\left( \pm \lambda_{1} \pm \lambda_{2} \pm \lambda_{3} \pm \lambda_{4}\right)$ where in first parenthesis have an even, in second parenthesis an odd number of negative signs, thus again $\rho_{3}+\rho_{4}$ is completely reducible. Finally, $\rho_{1}+\rho_{3}+\rho_{4}$ is completely reducible, since if $V$ is the module for $\rho_{1}, V^{\prime}$ the module for $\rho_{3}+\rho_{4}$, then $V^{\prime}=\check{V}^{\prime}$ and the weights of $V \otimes V^{\prime}$ are the weights of $\rho_{1} \otimes \rho_{3}, \rho_{1} \otimes \rho_{4}$, whence by above, never of the form $\pm a \alpha, \alpha \in \Delta$.

For $n=5$ the weights of $\rho_{2}$ are $\pm \lambda_{i} \pm \lambda_{j}(i \neq j)$ so it is self contragredient, whence $\rho_{1}+\rho_{5}$ seen to be completely reducible.

If the characteristic is two, $n=5, \rho_{2 A_{1}}$ has weights 2 weights of $\left.\rho_{1}\right\}$, so weights of $\rho_{2 \Lambda_{1}}+\rho_{4}$ (resp. $\rho_{2 \Lambda_{1}}+\rho_{5}$ ) are $\pm 2 \lambda_{i}+$ $1 / 2\left( \pm \lambda_{1} \pm \cdots \pm \lambda_{5}\right)$ odd (resp. even) number of negative signs, so once again it is completely reducible.

Type $E_{8}$. Here our notation differs from that of Bourbaki, with the labelling of the Dynkin diagram being

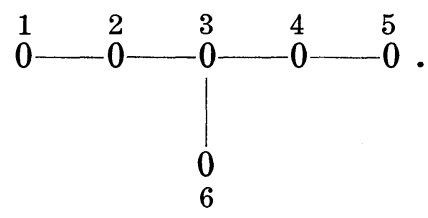

We need to investigate $\rho_{1}+\rho_{5}$.

The method used to find the weights of the spin and half spin representations cannot be used now, since there is no convenient description of the Weyl group for $E_{6}$. However, we can apply Springer's criterion [5], which involves only the highest weights $\Lambda_{1}, \Lambda_{5}$. We find $3\left(\Lambda_{5}+\rho, \Lambda_{5}+\rho\right)=135$ where $2 \rho=$ sum of the positive roots, hence for $p \neq 2 \rho_{1}+\rho_{5}$ is completely reducible.

Thus we have shown, by the above case by case examination that every representation listed in [2] is completely reducible, which if the characteristic is sufficiently large, shows all absolutely admissible representations to be completely reducible. 


\section{REFERENCES}

1. A. Borel, Linear Representations of semi-simple algebraic groups, Amer. Math. Soc., Proc. of Symposia in Pure Mathematics, 29 (1975), 421-440.

2. S. Haris, Admissible Representations for split reductive groups defined over a function field, Osaka J. Mato., 15 (1978), 101-116.

3. G. Hochschild, Cohomology of algebraic linear groups, Illinois J. Math., 5, number 3, (1961), 492-519.

4. J-I. Igusa, Geometry of absolutely admissible representations, Number Theory, Algebraic Geometry and Commutative Algebra, in honor of Y. Akizuki, Kinokuniya, Tokyo 1973, 373-452.

5. T. A. Springer, Weyl's Character Formula for Algebraic Groups, Inventiones Math., 5 (1968), 85-105.

6. G. Warner, Harmonic Analysis on Semi-Simple Lie Groups I, Springer-Verlag, Berlin 1972.

Received August 22, 1977. This work was partially supported by the National Science Foundation.

UNIVERSITY OF WASHINGTON

Seattle, WA 98195 


\section{PACIFIC JOURNAL OF MATHEMATICS}

\section{EDITORS}

RichaRd ARENS (Managing Editor)

University of California

Los Angeles, CA 90024

Charles W. Curtis

University of Oregon

Eugene, OR 97403

C. C. Moore

University of California

Berkeley, CA 94720

\section{J. DUGUNDJI}

Department of Mathematics

University of Southern California

Los Angeles, CA 90007

R. FinN and J. Milgram

Stanford University

Stanford, CA 94305

\section{E. F. BECKENBACH \\ B. H. NeumanN

\author{
UNIVERSITY OF BRITISH COLUMBIA \\ CALIFORNIA INSTITUTE OF TECHNOLOGY \\ UNIVERSITY OF CALIFORNIA \\ MONTANA STATE UNIVERSITY \\ UNIVERSITY OF NEVADA, RENO \\ NEW MEXICO STATE UNIVERSITY \\ OREGON STATE UNIVERSITY \\ UNIVERSITY OF OREGON
}

F. WOLF

K. Yoshida

\section{SUPPORTING INSTITUTIONS}

\author{
UNIVERSITY OF SOUTHERN CALIFORNIA \\ STANFORD UNIVERSITY \\ UNIVERSITY OF HAWAII \\ UNIVERSITY OF TOKYO \\ UNIVERSITY OF UTAH \\ WASHINGTON STATE UNIVERSITY \\ UNIVERSITY OF WASHINGTON
}

The Supporting Institutions listed above contribute to the cost of publication of this Journal, but they are not owners or publishers and have no responsibility for its content or policies.

Mathematical papers intended for publication in the Pacific Journal of Mathematics should be in typed form or offset-reproduced, (not dittoed), double spaced with large margins. Please do not use built up fractions in the text of the manuscript. However, you may use them in the displayed equations. Underline Greek letters in red, German in green, and script in blue. The first paragraph or two must be capable of being used separately as a synopsis of the entire paper. Items of the bibliography should not be cited there unless absolutely necessary, in which case they must be identified by author and journal, rather than by item number. Manuscripts, in triplicate, may be sent to any one of the editors. Please classify according to the scheme of Math. Reviews, Index to Vol. 39. All other communications should be addressed to the managing editor, or Elaine Barth, University of California, Los Angeles, California, 90024.

50 reprints to each author are provided free for each article, only if page charges have been substantially paid. Additional copies may be obtained at cost in multiples of 50 .

The Pacific Journal of Mathematics is issued monthly as of January 1966. Regular subscription rate: $\$ 72.00$ a year (6 Vols., 12 issues). Special rate: $\$ 36.00$ a year to individual members of supporting institutions.

Subscriptions, orders for numbers issued in the last three calendar years, and changes of address should be sent to Pacific Journal of Mathematics, P.O. Box 969, Carmel Valley, CA 93924, U.S.A. Older back numbers obtainable from Kraus Periodicals Co., Route 100, Millwood, NY 10546.

PUBLISHED BY PACIFIC JOURNAL OF MATHEMATICS, A NON-PROFIT CORPORATION

Printed at Kokusai Bunken Insatsusha (International Academic Printing Co., Ltd.). 8-8, 3-chome, Takadanobaba, Shinjuku-ku, Tokyo 160, Japan. 


\section{Pacific Journal of Mathematics \\ Vol. 79, No. $2 \quad$ June, 1978}

David R. Adams, Quasi-additivity and sets of finite $L^{p}$-capacity ........ 283

George M. Bergman and Warren Dicks, Universal derivations and universal

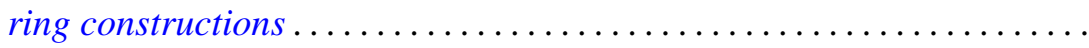

Robert F. Brown, Addendum to: "Fixed points of automorphisms of compact Lie groups".........................................

Eugene Frank Cornelius, Jr., Characterization of a class of torsion free

groups in terms of endomorphisms .......................

Andres del Junco, A simple measure-preserving transformation with trivial centralizer..................................... 357

Allan Lee Edmonds, Extending a branched covering over a handle ...... 363

Sjur Flam, A characterizaton of $\mathbf{R}^{2}$ by the concept of mild convexity .......

Claus Gerhardt, $L^{p}$-estimates for solutions to the instationary Navier-Stokes

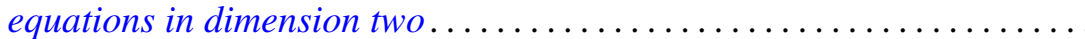

Kensaku Gomi, Finite groups with a standard subgroup isomorphic to

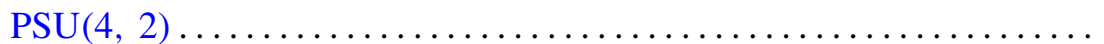

E. E. Guerin, A convolution related to Golomb's root function ........... 463

$\mathrm{H}$. B. Hamilton, Modularity of the congruence lattice of a commutative cancellative semigroup .................................

Stephen J. Haris, Complete reducibility of admissible representations over function fields.

Shigeru Itoh and Wataru Takahashi, The common fixed point theory of singlevalued mappings and multivalued mappings... ...

James E. Joseph, Multifunctions and graphs .............

Bruce Magurn, Images of $S K_{1} Z G$. .

Arnold Koster Pizer, A note on a conjecture of Hecke... .

Marlon C. Rayburn, Maps and h-normal spaces ...........

Barada K. Ray and Billy E. Rhoades, Corrections to: "Fixed-point theorems for mappings with a contractive iterate" ..............

Charles Irvin Vinsonhaler, Corrections to: "Torsion free abelian groups quasiprojective over their endomorphism rings. II". . . 